Effect of Gravity Correction on Isokinetic Average Force of the Quadriceps and Hamstring Muscle Groups in Women Runners

By: David H. Perrin, PhD, ATC , Esther M. Haskvitz, MS, PT, ATC, and Arthur Weltman, PhD*

Perrin, D. H., Haskvitz, E. M., \& Weltman, A. (1991). Effect of gravity correction on isokinetic average force of the quadriceps and hamstring muscle group in women runners. Isokinetics and Exercise Science, 1, 99-102.

***Note: Figures may be missing for this format of the document

***Note: Footnotes and endnotes indicated with brackets

\begin{abstract}
:
Previous investigations have examined the effect of gravity correction on hamstring and quadriceps force and reciprocal muscle group ratios when tested from a seated position. The effect of gravity correction on these values when knee extension is tested from supine and knee flexion from prone has not been determined. This study tested 48 females for concentric and eccentric average force of the quadriceps in the supine position and of the hamstring muscle group in the prone position with a Kin Com dynamometer. For the quadriceps and hamstring muscle groups, the gravity-corrected values were greater $(\mathrm{p}<.01)$ than gravity-uncorrected values for concentric and eccentric force. The gravity-corrected flexion/extension reciprocal muscle group ratios were also greater $(p<.01)$ than uncorrected ratios when determined from both concentric and eccentric average force values. These findings indicate gravity correction had a significant effect on both quadriceps and hamstring force production and reciprocal muscle group ratios, regardless of the fact that both muscle groups were assessed against the effects of gravity. Furthermore, the effect of gravity correction on the hamstring muscle group was approximately twice as great as on the quadriceps. These findings support the need to correct for the effect of gravity when assessing knee extension from supine and knee flexion from prone. Thus, it is very important for clinicians to report how testing and gravity correction were accomplished to allow proper interpretation and analysis of the presented values.
\end{abstract}

Article:

The importance of gravity correction in obtaining valid strength measures of the quadriceps and hamstring muscle groups when tested from the sitting position has been established.[3,7,8] Gravitational force tends to overestimate the strength of muscles assisted by gravity and to underestimate muscles opposed by gravity.5'6' 8 Depending on isokinetic test velocity, quadriceps force may be underpredicted by $4 \%[6]$ to $43 \%,[8]$ and hamstring force may be overpredicted by $15 \%[6]$ to $510 \%$.[8]

Failure to correct for the effects of gravity also confounds determination of the hamstring/quadriceps reciprocal muscle group ratio. Because gravity correction tends to reduce hamstring force and increase quadriceps force, determination of the reciprocal muscle group relationship from uncorrected data tends to inflate the ratio.[4,5]

Although isokinetic strength of the quadriceps and hamstring muscle groups has traditionally been assessed from the seated or supine position, recent research has suggested the sitting or supine positions may not be the optimal positions for assessment of hamstring strength, for several reasons. 
First, greater force is produced by the hamstring muscle group from the prone than the supine position.[2,9] Moreover, assessment of hamstring strength from the prone position, as well as quadriceps strength from the supine position, more closely approximates the length - tension relationship found during many sport activities.[9] Isokinetic testing from these positions occurs against the effect of gravity during both knee flexion and knee extension. However, the effect of gravity correction on force production and reciprocal muscle group ratios from these test positions has not been established. The purpose of this investigation was to determine the effects of gravity correction on concentric and eccentric average force of the quadriceps and hamstring muscle groups when assessed from the supine and prone positions, respectively. The effect of gravity correction on the hamstring/quadriceps reciprocal muscle group ratio was also determined.

\section{MATERIALS AND METHODS}

\section{Subjects}

Forty-eight females (age $28.3 \mathrm{yr}$, wt $59.5 \mathrm{~kg}$, ht $166.5 \mathrm{~cm}$ ) participated in the study after giving their informed consent in accordance with Institutional Human Investigation Committee guidelines. All subjects were free from history of injury to the lower extremity. Subjects were habitual runners (> $32 \mathrm{~km} / \mathrm{wk}$ ) who had been running for a minimum of 6 months.

\section{Instrumentation and Test Protocol}

Subjects were tested for isokinetic concentric and eccentric average force of the right side quadriceps and hamstring muscle groups at $45 \mathrm{deg} / \mathrm{sec}$ with a Kinetic Communicator (Kim Con) dynamometer (Chattecx, Inc., Chattanooga, TN). For assessment of the quadriceps muscle groups, each subject was placed supine on the dynamometer and stabilized with straps at the hip and knee. The range of motion tested was from -80 to $-20 \mathrm{deg}$ of knee extension. For assessment of the hamstring muscle group, subjects were placed prone on the dynamometer and stabilized with a strap at the hi p. The range of motion tested was from 20 to 80 deg of knee flexion.

For assessment of both knee extension and flexion average force, the Kin Com's force sensing pad was placed at the distal leg, and the axis of rotation of the dynamometer was aligned with the axis of rotation of the knee joint. Each subject performed a warm up session prior to measurement of both quadriceps and hamstring average force. The warmup consisted of two to five submaximal concentric and eccentric contractions. Following a brief rest period, subjects performed a minimum of three maximal contractions. Additional trials were performed, if necessary, to obtain three reproducible concentric and eccentric force curves, which were subsequently used for data analysis. A preload of 50 newtons was established for all test conditions.

\section{Gravity Correction Procedure}

Gravity correction procedures were followed prior to testing from both the supine and prone positions. In each instance, the limb was moved to an angle within the range of motion tested. This angle was then recorded relative to the horizontal plane, with above denoted as positive and below as negative. Subjects were then instructed to completely relax the limb on the loadcel 1 while the system measured the force due to gravity. Once this procedure was completed, it was then possible to retrieve either the gravity-corrected or - uncorrected force values from the Kin Com computer software.

\section{Data Analysis}

Values were obtained from the Kin Com software for both gravity-corrected and -uncorrected knee 
extension and flexion concentric and eccentric average force in newtons. Paired t-tests were then performed to determine if any differences existed between the gravity-corrected and -uncorrected values.

To determine the concentric and eccentric reciprocal muscle group ratios, the hamstring average force values were divided by the quadriceps values. Paired t-tests were then performed to determine if any differences existed between the reciprocal muscle group ratios derived from the gravitycorrected and -uncorrected average force values.

\section{RESULTS}

The means and standard deviations for both the gravity-corrected and -uncorrected average force values are presented in Table 1. For the quadriceps muscle group, the gravity-corrected values were $8.8 \%$ and $7.3 \%$ greater than the uncorrected values for concentric $(\mathrm{t}=31.79, \mathrm{df}=47, \mathrm{p}<.01)$ and eccentric $(\mathrm{t}=27.96, \mathrm{df}=47, \mathrm{p}<.01)$ average force, respectively. For the hamstring muscle group, the gravity-corrected values were $18.0 \%$ and $15.3 \%$ greater than the uncorrected values for concentric $(\mathrm{t}=31.27, \mathrm{df}=47, \mathrm{p}<.01)$ and eccentric $(\mathrm{t}=32.54, \mathrm{df}=47, \mathrm{p}<.01)$ average force, respectively.

The hamstring/quadriceps reciprocal muscle group ratios are presented in Table 2 . The gravitycorrected flexion/extension ratios were greater than the uncorrected ratios for both concentric $(\mathrm{t}=$ $7.25, \mathrm{df}=47, \mathrm{p}<.01)$ and eccentric $(\mathrm{t}=6.94, \mathrm{df}=47, \mathrm{p}<.01)$ average force.

\section{DISCUSSION}

The major finding of this investigation was that gravity correction had a significant effect on both concentric and eccentric quadriceps and hamstring muscle group force production and reciprocal muscle group ratios; even when both muscle groups were assessed against the effect of gravity. The influence of gravity correction on concentric strength of the quadriceps muscle group in our study was similar to other reports in the literature.[1,5] Appen and Duncan[1] and Fillyaw et al.[5] reported gravity-corrected quadriceps peak torque at $60 \mathrm{deg} / \mathrm{sec}$ was $4.5 \%$ and $5.2 \%$ greater than the uncorrected values respectively. Our slightly higher finding of $8.8 \%$ may be due to the greater absolute force generated from the slower test velocity $(45 \mathrm{deg} / \mathrm{sec})$.

We have been unable to find any reports examining the effect of gravity correction on eccentric strength of the quadriceps muscle group. Our findings indicate that although slightly less, the effect of gravity correction on eccentric force was essentially the same as that on concentric force for both the quadriceps and hamstring muscle groups (Table 1).

As expected, the effect of gravity correction on hamstring muscle group strength in our investigation (against gravity) was opposite to that found in reports which assessed hamstring strength from a seated position (gravity assisted). In particular, Appen and Duncan' and Fillyaw et al.5 reported respective decreases in hamstring peak torque of $12.6 \%$ and $16 \%$ when assessed at $60 \mathrm{deg} / \mathrm{sec}$. When hamstring strength was assessed from the prone position in our investigation, gravity correction resulted in increases of $18.0 \%$ and $15.3 \%$ for concentric and eccentric force, respectively. It is interesting to note that regardless of test position (prone or supine), gravity correction appears to have a much greater effect on hamstring strength than it does on quadriceps muscle group strength. This is probably due to the fact that the absolute strength generated by the hamstrings is considerably less than that produced by the quadriceps muscle group. As such, the amount of torque 
or force added to or detracted from the hamstrings as a result of gravity correction is a greater percentage of the peak (or average) values than for the stronger quadriceps. This point can be further illustrated by the force-velocity relationship. Appen and Duncan' assessed the effect of gravity at 60, 180,240 , and $300 \mathrm{deg} / \mathrm{sec}$. As concentric peak torque decreased with increases in test velocity, the effect of gravity correction became more pronounced. For the quadriceps muscle group, the gravitycorrected values were $4.5 \%$ greater than uncorrected values at $60 \mathrm{deg} / \mathrm{sec}$, but were $14.1 \%$ greater at $300 \mathrm{deg} / \mathrm{sec}$. Gravity-corrected hamstring values were $12.6 \%$ and $15.8 \%$ greater than uncorrected values at 60 and $300 \mathrm{deg} / \mathrm{sec}$, respectively.

The influence of gravity correction on the hamstring/quadriceps reciprocal muscle group ratio when strength is assessed from the seated position is well documented. "4.5 Gravity correction adds to the quadriceps value and detracts from the hamstring value, which tends to decrease the hamstring/quadriceps reciprocal muscle group ratio. In contrast, gravity correction tends to increase this ratio when quadriceps strength is assessed from supine and hamstring strength from prone. The magnitude of this effect on the ratio was slightly less in our investigation (6\% greater) than found by Appen and Duncan' (10\% less) and Fillyaw et al.5 (19.5\% less).

The hamstring/quadriceps reciprocal muscle group ratios determined from the eccentric average force values were essentially the same as those derived from the concentric values. Moreover, the effect of gravity correction was essentially the same for the ratios determined from the concentric and eccentric quadriceps and hamstring force values (Table 2).

Table 2 Gravity uncorrected (GUC) and gravity corrected $(\mathrm{GC})$ concentric and eccentric hamstring/quadriceps reciprocal muscle group ratios.

\begin{tabular}{lcc}
\hline & Concentric & Eccentric \\
\hline GUC & .51 & .51 \\
GC & .57 & .56 \\
\hline
\end{tabular}

The findings of this investigation indicate that gravity correction has a significant effect on both quadriceps and hamstring muscle group force production and reciprocal muscle group ratios, regardless of the fact that both muscle groups are assessed against the effect of gravity. The effect of gravity correction on the hamstring muscle group is approximately twice as great as that on the quadriceps. Since greater hamstring force can be generated from the prone position, clinicians are advised to consider this test position, and to obtain force and reciprocal muscle group ratios from gravity- corrected data. Appropriate reporting of isokinetic data requires clinicians to indicate test position and, if gravity corrected, how correction was achieved, thus allowing interpretation and replication. 


\section{REFERENCES}

Appen L, Duncan PW: Strength relationship of the knee musculature: effects of gravity and sport. J Orthop Sports Phys Ther 7:232- 235, 1986.

Barr AE, Duncan PW: Influence of position on knee flexor peak torque. J Orthop Sports Phys Ther 9:279-283, 1988.

Bohannon RW, Smith MB: Intrasession reliability of angle specific knee extension torque measurements with gravity corrections. J Orthop Sports Phys Ther 11:155- 157, 1989

Figoni SF, Christ CB, Massey BH: Effects of speed, hip and knee angle, and gravity on hamstring to quadriceps torque ratios. J Orthop Sports Phys Ther 9:287-291, 1988.

Fillyaw M, Bevins T, Fernandez L: Importance of correcting isokinetic peak torque for the effect of gravity when calculating knee flexor to extensor muscle ratios. Phys Ther 66:23-29, 1986.

Nelson SG, Duncan PW: Correction of isokinetic and isometric torque recordings for the effects of gravity. Phys Ther 63:674-676, 1983.

Rothstein JM, Lamb RL, Mayhew TP: Clinical uses of isokinetic measurements. Phys Ther 67:1840-1844, 1987.

Winter DA, Wells RP, Orr GW: Errors in the use of isokinetic dynamometers. Eup J Appl Physiol 46:397- 408, 1981.

9. Worrell TW, Denegar CR, Armstrong SL, Perrin DH: Effect of body position on hamstring muscle group average torque. J Orthop Sports Phys Ther 11:449-452, 1990. 